\title{
Texture features of periaqueductal gray in the patients with medication-overuse headache
}

\author{
Zhiye Chen ${ }^{1,2,3}$, Xiaoyan Chen ${ }^{2}$, Mengqi Liu ${ }^{1,3}$, Shuangfeng Liư ${ }^{1}$, Lin Ma ${ }^{1 *}$ and Shengyuan $\mathrm{Yu}^{2^{*}}$
}

\begin{abstract}
Background: Periaqueductal gray (PAG) is the descending pain modulatory center, and PAG dysfunction had been recognized in migraine. Here we propose to investigate altered PAG texture features (quantitative approach for extracting texture descriptors for images) in the patients with medication-overuse headache (MOH) based on high resolution brain structural image to understand the $\mathrm{MOH}$ pathogenesis.
\end{abstract}

Methods: The brain structural images were obtained from 32 normal controls (NC) and $44 \mathrm{MOH}$ patients on $3.0 \mathrm{~T}$ MR system. PAG template was created based on the ICBM152 gray matter template, and the individual PAG segment was performed by applying the deformation field to the PAG template after structural image segment. Grey-level co-occurrence matrix (GLCM) was performed to measure the texture parameters including angular second moment (ASM), Contrast, Correlation, inverse difference moment (IDM) and Entropy.

Results: Contrast was increased in $\mathrm{MOH}$ patients $(9.28 \pm 3.11)$ compared with that in NC $(7.94 \pm 0.65)(P<0.05)$, and other texture features showed no significant difference between $\mathrm{MOH}$ and $\mathrm{NC}(P>0.05)$. The area under the ROC curve was 0.697 for Contrast in the distinction of $\mathrm{MOH}$ from NC, and the cut-off value of Contrast was 8.11 with sensitivity 70 . $5 \%$ and specificity $62.5 \%$. The contrast was negatively with the sleep scores $(r=-0.434, P=0.003)$.

Conclusion: Texture Contrast could be used to identify the altered MR imaging characteristics in $\mathrm{MOH}$ in understanding the $\mathrm{MOH}$ pathogenesis, and it could also be considered as imaging biomarker in for $\mathrm{MOH}$ diagnosis.

Keywords: Brain, Magnetic resonance imaging, Medication-overuse headache, Migraine, Periaqueductal gray, Texture analysis

\section{Background}

Periaqueductal gray (PAG) is a center with powerful descending pain modulatory center in the midbrain, which include various layered neurons around the aquaeductus mesencephali $[1,2]$, and whose dysfunction had been recognized in migraine [3]. PAG, as a substantial descending pain modulatory center, exerts inhibition and facilitation control on nociceptive transmission in the dorsal horn and trigeminal nucleus [4], and the modulatory mechanism was exerted by descending PAG-RVM (rostral ventromedial medulla) pathway

\footnotetext{
* Correspondence: cjr.malin@vip.163.com; yusy1963@126.com

'Department of Radiology, Chinese PLA General Hospital, Beijing 100853,

China

2Department of Neurology, Chinese PLA General Hospital, Beijing 100853,

China

Full list of author information is available at the end of the article
}

contributing to central sensitization and development of secondary hyperalgesia [4, 5]. PAG included multiple types of neurons (eg. L-glutamate, $\gamma$-aminobutyric acid (GABA), opioids (particularly enkephalin), substance P), and had distinct connections with the forebrain, brainstem, and nociceptive neurons of lamina I of the spinal cord and trigeminal nucleus [6-9]. Therefore, PAG was confirmed as a critical component of a network responsing to pain and receiving functionally input from nociceptive pathways [10-12].

In the previous studies, the specific PAG lesions had been identified in multiple sclerosis [13-17] and infarction [18], and nonspecific PAG lesions was also revealed in episodic migraine (EM) patients in our previous study [19]. The specific lesions was the direct evidence for migraine, and the nonspecific lesions was indirectly used to explain 
the migraine pathogenesis, which may be associated with iron deposition and may be considered as a possible "generator" of migraine attacks [1, 20, 21]. However, the neuromechinism for nonspecific PAG lesions in migraine was still not elucidated up to now.

Medication-overuse headache $(\mathrm{MOH})$ is a secondary form of chronic headache deriving from episodic migraine (EM) related to the overuse of triptans, analgesics and other acute headache medications [22-24]. Resting-state functional MRI (rs-fMRI) demonstrated altered functional connectivity was revealed in $\mathrm{MOH}$, and suggested that $\mathrm{MOH}$ is associated with intrinsic brain network changes rather with macrostructural changes [23]. Voxel-based morphometry (VBM) recognized that increased gray matter in the midbrain presented in $\mathrm{MOH}$ [25]. Recently, some studies also confirmed an altered nucleus accumbens functional connectivity of motivational circuits [22] and abnormal connectivity between the PAG and other pain modulatory (frontal) regions in $\mathrm{MOH}$, which were consistent with dysfunctional central pain control [26]. Although the functional and structural MRI recognized the PAG dysfunction in $\mathrm{MOH}$ patients, these methods did not presented the detailed changes of the intrinsic natures of PAG in MOH patients.

Texture features are the intrinsic properties of image and provide an efficient image classification to detect subthe alterations in the gray level distribution of an image [27]. Texture feature analysis had been widely applied in the brain tumor [28, 29], epilepsy [30,31], muscular dystrophy [32], Attention-Deficit/Hyperactivity Disorder classification [33], and mild cognitive impairment [34]. However, MR imaging texture feature analysis was not applied in medication-overuse headache $(\mathrm{MOH})$ so far.

In this study, we hypothesize MOH patients without T2visible lesions may present altered texture features changes in MR structural images. To address this hypothesis, we prospectively obtained high resolution structural images from $44 \mathrm{MOH}$ patients and $32 \mathrm{NCs}$ without T2-visible lesions on the brain. Gray level co-occurrence matrix(GLCM) $[35,36]$ was used to calculate the texture parameters of PAG including angular second moment (ASM), Contrast, Correlation, inverse difference moment (IDM) and Entropy in the subjects, which would be used to detect the texture features change for PAG to elucidate the neuromechnism of PAG dysfunction in $\mathrm{MOH}$ pathogenesis.

\section{Methods}

\section{Subjects}

Written informed consent was obtained from all participants according to the approval of the ethics committee of the local institutional review board. Forty-four $\mathrm{MOH}$ patients and 32 normal controls were recruited from the International Headache Center, Department of Neurology, Chinese PLA General Hospital. All the following inclusion criteria should be fulfilled: 1) MOH refers to ICHD-III beta 8.2, and the definition of migraine refers to ICHD-III beta 1.1 and 1.2 [37]; 2) no migraine preventive medication used in the past 3 months; 3 ) age between 20 and 60 years; 4) right-handed; 5) absence of any chronic disorders, including hypertension, hypercholesterolemia, diabetes mellitus, cardiovascular diseases, cerebrovascular disorders, neoplastic diseases, infectious diseases, connective tissue diseases, other subtypes of headache, chronic pain other than headache, severe anxiety or depression preceding the onset of headache, psychiatric diseases, etc.; 6) absence of alcohol, nicotine, or other substance abuse; and 7) patient's willingness to engage in the study. Thirty-two normal controls (NCs) were recruited from the hospital's staff and their relatives. Inclusion criteria were similar to those of patients, except for the first two items, and NCs should never have had any primary headache disorders or other types of headache in the past year. The exclusion criteria for $\mathrm{NC}$ and $\mathrm{MOH}$ were the following: cranium trauma, illness interfering with central nervous system function, psychotic disorder, and regular use of a psychoactive or hormone medication. General demographic and headache information were registered and evaluated in our headache database. Additionally, we evaluated anxiety, depression, and cognitive function of all the participants by using the Hamilton Anxiety Scale (HAMA) [38], the Hamilton Depression Scale (HAMD) [39], and the Montreal Cognitive Assessment (MoCA) Beijing Version (www.mocatest.org). All the patients were given with the Visual Analogue Scale (VAS), migraine disability assessment (MIDSA), and a standard categorical four-grade sleep disturbance scale (SDS)(0, normal; 1, mild sleep disturbance; 2, moderate sleep disturbance; 3, serious sleep disturbance). MRI scans were taken in the interictal stage at least three days after a migraine attack for $\mathrm{MOH}$ patients. All the subjects were right-handed and underwent conventional MRI examination to exclude the subjects with cerebral infarction, malacia, or occupying lesions. Alcohol, nicotine, caffeine, and other substances were avoided for at least $12 \mathrm{~h}$ before MRI examination.

\section{MRI acquisition}

Images were acquired on a GE 3.0 T MR system (DISCOVERY MR750, GE Healthcare, Milwaukee, WI, USA) and a conventional eight-channel quadrature head coil was used. All subjects were instructed to lie in a supine position, and formed padding was used to limit head movement. A three-dimensional T1-weighted fast spoiled gradient recalled echo (3D T1-FSPGR) sequence generating 180 contiguous axial slices [TR (repetition time) $=6.3 \mathrm{~ms}$, TE (echo time) $=2.8 \mathrm{~ms}$, flip angle $=15^{\circ}$, FOV (field of view) $=25.6 \mathrm{~cm} \times 25.6 \mathrm{~cm}$, Matrix $=256 \times$ 256, NEX (number of acquisition) $=1$ ] was used to perform 


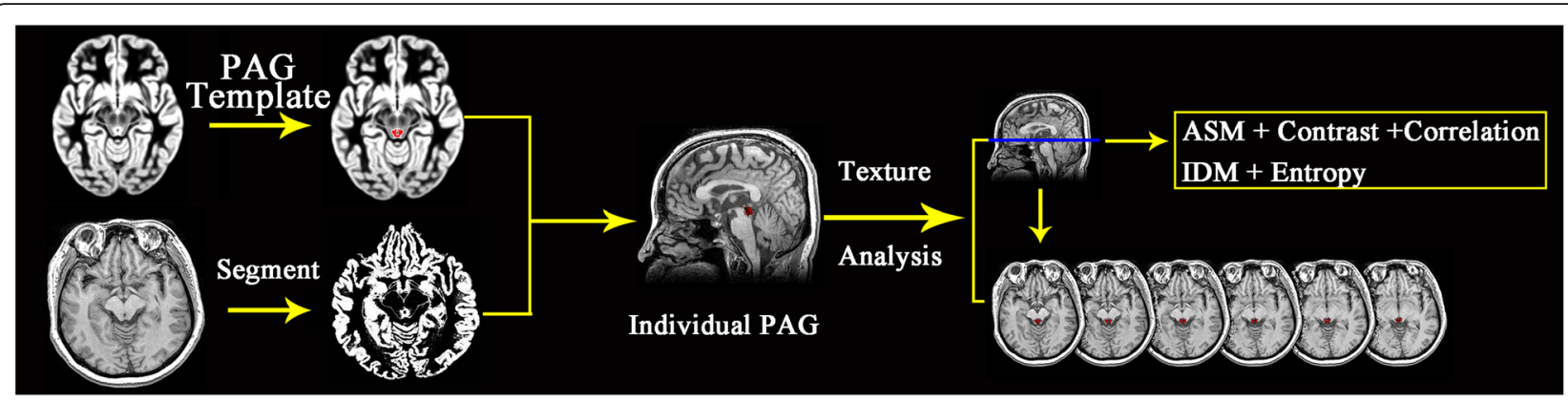

Fig. 1 The flowchart of PAG texture calculation. GLCM, grey-level co-occurrence matrix; ASM, Angular Second Moment; IDM, Inverse Difference Moment

the new segment and the individual PAG creation. Conventional T2-weighted imaging (T2WI), T1 fluid-attenuated inversion recovery (T1-FLAIR) and diffusion weighted imaging (DWI) were also acquired. All imaging protocols were identical for all subjects. No obvious structural damage and T2-visible lesion were observed on the conventional MR images.

\section{MR image processing}

All MR structural image data were processed using Statistical Parametric Mapping 12 (SPM12) (http:// www.fil.ion.ucl.ac.uk/spm/) running under MATLAB 7.6 (The Mathworks, Natick, MA, USA) to perform segment [40]. The image processing included following steps: (1) Create PAG template based on mni_icbm152_gm_tal_nlin_asym_09a template using MRIcron software (http://people.cas.sc.edu/rorden/mricron/index.html); (2) The structural images segment were performed with the new segment tool of SPM12 software, and the deformation field(iy_subjectid.nii) was generated. (3) Individual PAG mask (wPAG.nii) was generated by apply the deformation field (generated by new segment) to the PAG template using deformations tool of SPM12 software; (4) The individual PAG(wPAG_AddMask.nii) were segmented by an in-house script written on MATLAB (the Math Works, Inc., Natick, MA, USA) platform. The inhouse script was provided in the in Additional file 1. (5) The PAG texture parameters were calculated over the whole PAG using gray-level co-occurrence matrix(GLCM) with the GLCM plugins on ImagJ(1.50i)(https://imagej .nih.gov/ij). The texture parameters included ASM, Contrast, Correlation, IDM and Entropy [35, 41] (Fig. 1). These texture parameters were measured on each slice, and the mean texture parameters values over all the slices were regarded as the final texture parameter value.

\section{Statistical analysis}

The statistical analysis was performed by using PASW Statistics 18.0. Independent sample $T$ test was applied to age, HAMA, HAMD, MoCA scores and all the texture parameters. Chi-Square test was applied to sex. Pearson correlation analysis was applied between Contrast and the clinical variables. Significant difference was set at a $P$ value of $<0.05$. Receiver operating characteristics (ROC) curve analysis was applied to evaluate the diagnostic efficacy of Contrast.

\section{Results}

Demography and neuropsychological test

There was no significant difference for age and sex between $\mathrm{MOH}$ and $\mathrm{NC}$, a significant difference for HAMA between $\mathrm{MOH}(18.25 \pm 8.74)$ and $\mathrm{NC}(10.19 \pm 2.98)$, HAMD between $\mathrm{MOH}(19.80 \pm 11.85)$ and $\mathrm{NC}(8.03 \pm$ 4.34), MoCA among MOH (23.43 \pm 3.72$)$ and NC (27.16 \pm 2.32 ) (Table 1).

\section{Comparison of PAG texture parameters between $\mathrm{MOH}$ and NC}

Table 2 demonstrated that there was a significant increased Contrast in $\mathrm{MOH}(9.28 \pm 3.11)$ compared with that in NC $(7.94 \pm 0.65)(P<0.05)$. The ASM, Correlation,

Table 1 The clinical characteristics of normal controls and $\mathrm{MOH}$ patients

\begin{tabular}{lllll}
\hline & $\mathrm{NC}$ & $\mathrm{MOH}$ & T value & $P$ value \\
\hline Sex(M/F) & $32(12 / 20)$ & $44(9 / 35)$ & $2.692^{\mathrm{a}}$ & 0.101 \\
Age(year) & $41.34 \pm 10.89$ & $42.30 \pm 9.62$ & 0.403 & 0.688 \\
HAMA & $10.19 \pm 2.98$ & $18.25 \pm 8.74$ & 5.002 & 0.000 \\
HAMD & $8.03 \pm 4.34$ & $19.80 \pm 11.85$ & 5.354 & 0.000 \\
MoCA & $27.16 \pm 2.32$ & $23.43 \pm 3.72$ & 4.999 & 0.000 \\
DD(year) & $11.25 \pm 9.30$ & & & \\
VAS & $7.88 \pm 1.45$ & & & \\
MIDSA & $101.81 \pm 53.95$ & & & \\
Frequence(month) & $24.81 \pm 6.32$ & & & \\
SDS & $2.23 \pm 1.36$ & & & \\
\hline
\end{tabular}

aChi-Square test; $N C$ normal control, $\mathrm{MOH}$ medication-overuse headache, $D D$ disease duration, VAS visual analogue scale; HAMA Hamilton Anxiety Scale, HAMD Hamilton Depression Scale, MoCA Montreal Cognitive Assessment, NA not available, SDS standard categorical four-grade sleep disturbance scale (0, normal; 1 , mild sleep disturbance; 2 , moderate sleep disturbance; 3 , serious sleep disturbance) 
Table 2 Comparison of PAG texture parameters among $\mathrm{NC}$ and $\mathrm{MOH}$

\begin{tabular}{lcccc}
\hline & NC & MOH & T value & $P$ value \\
\hline ASM $\left(\times 10^{-3}\right)$ & $0.998768 \pm 0.000124$ & $0.998709 \pm 0.000175$ & 1.656 & 0.102 \\
Contrast & $7.943417 \pm 0.645233$ & $9.282469 \pm 3.109250992$ & 2.395 & 0.019 \\
Correlation & $0.06879277 \pm 0.01808873$ & $0.062252806 \pm 0.030849871$ & 1.072 & 0.287 \\
$\operatorname{IDM}\left(\times 10^{-3}\right)$ & $0.999345 \pm 0.000051$ & $0.999331992 \pm 0.0000595448$ & 1.007 & 0.317 \\
Entropy $\left(\times 10^{-5}\right)$ & $0.008454 \pm 0.000705$ & $0.008718528 \pm 0.00096365$ & 1.317 & 0.192 \\
\hline
\end{tabular}

ASM Angular Second Moment, IDM Inverse Difference Moment

IDM and Entropy showed no significant difference between $\mathrm{MOH}$ and NC. Figure 2 presented the distribution of increased Contrast in $\mathrm{MOH}$ patients, and the other texture parameters showed no significant change in $\mathrm{MOH}$ patients compared with $\mathrm{NC}$ patients.

\section{ROC curve analysis and correlation analysis for Contrast} ROC analysis demonstrated that area under curve (AUC) of Contrast was 0.697 in $\mathrm{NC}$ vs. $\mathrm{MOH}$, and the cut-off value was 8.11 with sensitivity $70.5 \%$ and specificity $62.5 \%$ (Fig. 3 ). The contrast was negatively with the SDS scores $(r=-0.434, P=0.003)$, and presented no significant correlation with other clinical variables.

\section{Discussion}

GLCM has proved to be a popular statistical method of extracting textural feature from MR images. In this study, five texture features were extracted, and previous study recognized that ASM represented the image energy, IDM represented the local homogeneity, Entropy represented the amount of information of the image that is needed for the image compression, and Correlation represented the linear dependency of grey levels of neighboring pixels [42]. In the current study, these four texture features did not showed significant difference between $\mathrm{MOH}$ patients and NCs, which suggested these four texture features could not elucidate the $\mathrm{MOH}$ pathogenesis, and they were not considered as diagnostic variables.

Contrast represents the amount of local gray level variation in an image, and a high value high value of this parameter may indicate the presence of noise or "wrinkled" textures in the image [30]. In this study, the increased Contrast texture parameter was identified in $\mathrm{MOH}$ patients, which suggested that PAG present increased local gray level variation in MR T1 images. The increased noise in PAG image may be associated with local heterogeneous intensity, which may be influenced by the iron deposition $[20,21]$ or other factors, and the neuromechanism should be further investigated.

Further ROC analysis revealed that AUC was 0.697 for Contrast, and the cut-off value (8.11) presented with sensitivity $70.5 \%$ and specificity $62.5 \%$, which indicated that Contrast might be consider as an imaging biomarker for the diagnosis of $\mathrm{MOH}$. Correlation analysis demonstrated that Contrast was negatively related to SDS scores, which indicated that decreased sleep quality was associated with the $\mathrm{MOH}$ pathogenesis. And other clinical variables showed no any correlation with Contrast, and these findings demonstrated that neuropsychological factors and pain intensity may be not associated with the PAG dysfunction in $\mathrm{MOH}$ pathogenesis.

In this study, GLCM method was used to calculate the MR image texture features on $\mathrm{MOH}$ patients, and texture Contrast was screened from the five texture parameters to explain the PAG dysfunction in $\mathrm{MOH}$ patients. However, there were several limitations in our study. Firstly, this study was based on GLCM method to calculate the texture features of PAG, and the other novel texture analysis methods such as histogram analysis and first order texture analysis should be considered in the future. Secondly, only five texture features were calculated in this study, and more texture features should be measured to screen the significant texture features for $\mathrm{MOH}$ patients. Lastly, 3D high resolution structural image was used to calculate the PAG texture features because of its high contrast for PAG, and the other MR images such as T2 weighted image and susceptibility
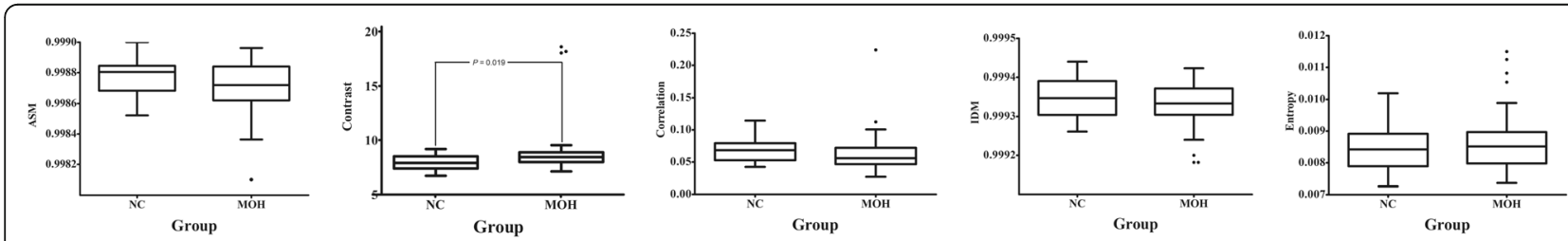

Fig. 2 Comparison of PAG texture between $\mathrm{MOH}$ and $\mathrm{NC}$, and the significant difference of Contrast presented in $\mathrm{MOH}$ compared with $\mathrm{NC}$ 


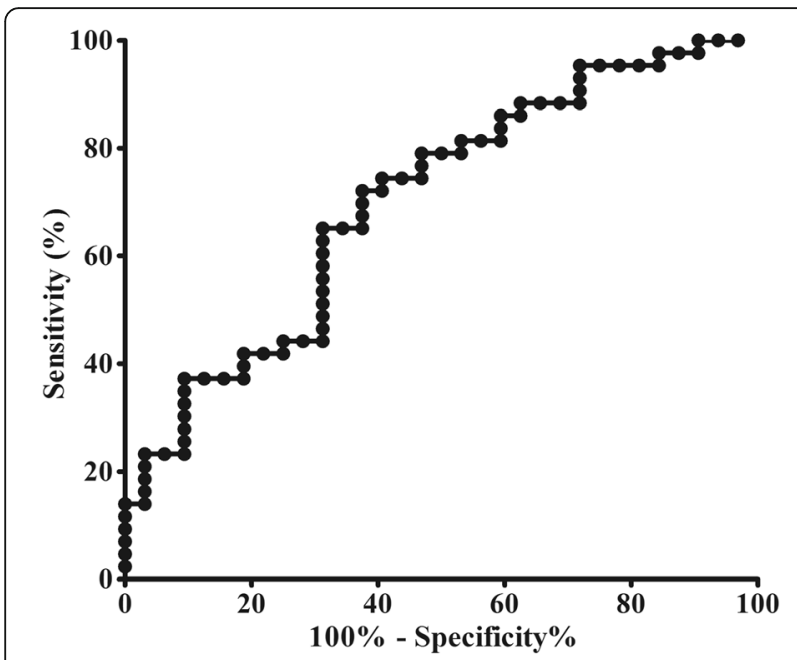

Fig. 3 ROC curve for Contrast to diagnose MOH from NC. The area under the curve was 0.697, and the cut-off value of Contrast was 8.11 with sensitivity $70.5 \%$ and specificity $62.5 \%$

weighted image should also be considered for the texture analysis in the future.

\section{Conclusion}

In conclusion, this study revealed that altered PAG texture Contrast presented in $\mathrm{MOH}$ patients undetected by visual assessment, and it may be associated with PAG dysfunction and may be considered as a auxiliary diagnostic and evaluated imaging biomarker in $\mathrm{MOH}$ patients.

\section{Additional file}

Additional file 1: The in-house script written on MATLAB (the Math Works, Inc., Natick, MA, USA) platform was used to segment individual PAG. (DOC $22 \mathrm{~kb})$

\section{Abbreviations}

ASM: angular second moment; GLCM: grey-level co-occurrence matrix; IDM: inverse difference moment; $\mathrm{MOH}$ : medication-overuse headache; NC: normal controls; PAG: periaqueductal gray

\section{Acknowledgments}

This work was supported by the National Natural Sciences Foundation of China (81371514), the Special Financial Grant from the China Postdoctoral Science Foundation (2014 T70960) and the Foundation for Medical and health Sci \& Tech innovation Project of Sanya (2016YW37).

\section{Authors' contributions}

Category 1: (a) Conception and Design: L. M; SY. Y. (b) Acquisition of Data: ZY. C; MQ. L; SF. L; XY. C. (c) Analysis and Interpretation of Data: ZY. C. Category 2: (a) Drafting the Article: ZY. C. (b) Revising It for Intellectual Content: L. M; SY. Y. All authors read and approved the final manuscript.

\section{Competing interests}

The authors declare that they have no competing interests.

\section{Author details}

'Department of Radiology, Chinese PLA General Hospital, Beijing 100853, China. ${ }^{2}$ Department of Neurology, Chinese PLA General Hospital, Beijing
100853, China. ${ }^{3}$ Department of Radiology, Hainan Branch of Chinese PLA General Hospital, Beijing 100853, China.

Received: 18 January 2017 Accepted: 20 January 2017

Published online: 02 February 2017

\section{References}

1. Welch KM, Nagesh V, Aurora SK, Gelman N (2001) Periaqueductal gray matter dysfunction in migraine: cause or the burden of illness? Headache 41:629-37

2. Smith GS, Savery D, Marden C, Lopez Costa JJ, Averill S, Priestley JV, Rattray M (1994) Distribution of messenger RNAs encoding enkephalin, substance $P$, somatostatin, galanin, vasoactive intestinal polypeptide, neuropeptide $Y$, and calcitonin gene-related peptide in the midbrain periaqueductal grey in the rat. J Comp Neurol 350:23-40

3. Raskin NH, Yoshio H, Sharon L (1987) Headache may arise from perturbation of brain. Headache 27:416-20

4. Heinricher MM, Tavares I, Leith JL, Lumb BM (2009) Descending control of nociception: specificity, recruitment and plasticity. Brain Res Rev 60:214-25

5. Fields H (2004) State-dependent opioid control of pain. Nat Rev Neurosci 5: 565-75

6. Benarroch EE (2012) Periaqueductal gray: an interface for behavioral control. Neurology 78:210-7

7. An X, Bandler R, Ongür D, Price JL (1998) Prefrontal cortical projections to longitudinal columns in the midbrain periaqueductal gray in Macaque monkeys. J Comp Neurol 401:455-79

8. Herbert H, Saper CB (1992) Organization of medullary adrenergic and noradrenergic projections to the periaqueductal gray matter in the rat. J Comp Neurol 315:34-52

9. Yezierski RP (1988) Spinomesencephalic tract: projections from the lumbosacral spinal cord of the rat, cat, and monkey. J Comp Neurol 267:131-46

10. Keay KA, Bandler R (2002) Distinct central representations of inescapable and escapable pain: observations and speculation. Exp Physiol 87:275-9

11. Parry DM, Macmillan FM, Koutsikou S, Mcmullan S, Lumb BM (2008) Separation of A- versus C-nociceptive inputs into spinal-brainstem circuits. Neuroscience 152:1076-85

12. Lumb BM (2004) Hypothalamic and midbrain circuitry that distinguishes between escapable and inescapable pain. News Physiol Sci 19:22-6

13. Gee JR, Chang J, Dublin AB, Vijayan N (2005) The association of brainstem lesions with migraine-like headache: an imaging study of multiple sclerosis. Headache 45:670-7

14. Haas DC, Kent PF, Friedman DI (1993) Headache caused by a single lesion of multiple sclerosis in the periaqueductal gray area. Headache 33:452-5

15. Lin GY, Wang CW, Chiang TT, Peng GS, Yang FC (2013) Multiple sclerosis presenting initially with a worsening of migraine symptoms. J Headache Pain 14:70

16. Tortorella P, Rocca MA, Colombo B, Annovazzi P, Comi G, Filippi M (2006) Assessment of MRI abnormalities of the brainstem from patients with migraine and multiple sclerosis. J Neurol Sci 244:137-41

17. Fragoso YD, Brooks JB (2007) Two cases of lesions in brainstem in multiple sclerosis and refractory migraine. Headache 47:852-4

18. Wang Y, Wang XS (2013) Migraine-like headache from an infarction in the periaqueductal gray area of the midbrain. Pain Med 14:948-9

19. Chen Z, Chen X, Liu M, Liu S, Ma L, Yu S (2016) Nonspecific periaqueductal gray lesions on T2WI in episodic migraine. J Headache Pain 17:101

20. Kruit MC, Launer LJ, Overbosch J, van Buchem MA, Ferrari MD (2009) Iron accumulation in deep brain nuclei in migraine: a population-based magnetic resonance imaging study. Cephalalgia 29:351-9

21. Tepper SJ, Lowe MJ, Beall E, Phillips MD, Liu K, Stillman MJ et al (2012) Iron deposition in pain-regulatory nuclei in episodic migraine and chronic daily headache by MRI. Headache 52:236-43

22. Torta DM, Costa T, Luda E, Barisone MG, Palmisano P, Duca S et al (2016) Nucleus accumbens functional connectivity discriminates medication-overuse headache. Neuroimage Clin 11:686-93

23. Chanraud S, Di Scala G, Dilharreguy B, Schoenen J, Allard M, Radat F (2014) Brain functional connectivity and morphology changes in medication-overuse headache: Clue for dependence-related processes? Cephalalgia 34:605-15

24. Tepper SJ (2012) Medication-overuse headache. Continuum (Minneap Minn) 18:807-22

25. Riederer F, Gantenbein AR, Marti M, Luechinger R, Kollias S, Sandor PS (2013) Decrease of gray matter volume in the midbrain is associated with 
treatment response in medication-overuse headache: possible influence of orbitofrontal cortex. J Neurosci 33:15343-9

26. Michels L, Christidi F, Steiger VR, Sandor PS, Gantenbein AR, Landmann G, et al (2016) Pain modulation is affected differently in medication-overuse headache and chronic myofascial pain - A multimodal MRI study. Cephalalgia. doi:10.1177/0333102416652625

27. Herlidoumême $S$, Constans JM, Carsin B, Olivie D, Eliat PA, Nadaldesbarats L et al (2003) MRI texture analysis on texture test objects, normal brain and intracranial tumors. Magn Reson Imaging 21:989-93

28. Nachimuthu DS, Baladhandapani A (2014) Multidimensional texture characterization: on analysis for brain tumor tissues using MRS and MRI. J Digit Imaging 27:73-81(9)

29. Mahmoud-Ghoneim D, Toussaint G, Constans JM, de Certaines JD (2003) Three dimensional texture analysis in MRl: a preliminary evaluation in gliomas. Magn Reson Imaging 21:983-7

30. Oliveira MSD, Betting LE, Mory SB, Cendes F, Castellano G (2013) Texture analysis of magnetic resonance images of patients with juvenile myoclonic epilepsy. Epilepsy Behav 27:22-8

31. Caselato GR, Kobayashi E, Bonilha L, Castellano G, Rigas AH, Li LM et al (2003) Hippocampal texture analysis in patients with familial mesial temporal lobe epilepsy. Arq Neuropsiquiatr 61(Suppl 1):83-7

32. Certaines JDD, Larcher T, Duda D, Azzabou N, Eliat PA, Escudero LM et al (2015) Application of texture analysis to muscle MRI: 1-What kind of information should be expected from texture analysis? EPJ Nonlinear Biomed Phys 3:1-14

33. Chang CW, Ho CC, Chen JH (2012) ADHD classification by a texture analysis of anatomical brain MRI data. Front Syst Neurosci 6:66

34. de Oliveira MS, Balthazar ML, D'Abreu A, Yasuda CL, Damasceno BP, Cendes F, Castellano G (2011) MR imaging texture analysis of the corpus callosum and thalamus in amnestic mild cognitive impairment and mild Alzheimer disease. AJNR Am J Neuroradiol 32:60-6

35. Haralick RM, Shanmugam K, Dinstein IH (1973) Textural Features for Image Classification. IEEE Trans Syst Man Cybern smc-3:610-21

36. Rajkovic N, Kolarevic D, Kanjer K, Milosevic NT, Nikolic-Vukosavljevic D, Radulovic M (2016) Comparison of Monofractal, Multifractal and gray level Co-occurrence matrix algorithms in analysis of Breast tumor microscopic images for prognosis of distant metastasis risk. Biomed Microdevices 18:83

37. Headache Classification Committee of the International Headache Society (IHS) (2013) The International Classification of Headache Disorders, 3rd edition (beta version). Cephalalgia 33:629-808.

38. Maier W, Buller R, Philipp M, Heuser I (1988) The Hamilton Anxiety Scale: reliability, validity and sensitivity to change in anxiety and depressive disorders. J Affect Disord 14:61-8

39. Hamilton M (1967) Development of a rating scale for primary depressive illness. Br J Soc Clin Psychol 6:278-96

40. Ashburner J, Friston KJ (2000) Voxel-based morphometry-the methods. Neuroimage 11:805-21

41. Walker RF, Jackway P, Longstaff ID (1995) Improving Co-occurrence Matrix Feature Discrimination. DICTA 3rd Conference on Digital Image Computing: Techniques and Application. Brisbane: University of Queensland, 643-648

42. Mohanaiah P, Sathyanarayana P, Gurukumar L (2014) Image texture feature extraction using GLCM approach. Int J Sci Res Publ 3:1-5

\section{Submit your manuscript to a SpringerOpen ${ }^{\circ}$ journal and benefit from:}

- Convenient online submission

- Rigorous peer review

- Immediate publication on acceptance

- Open access: articles freely available online

- High visibility within the field

- Retaining the copyright to your article 